\title{
Approvisionnement Et Commercialisation De Deux Espèces De Francolin Utilisées Comme Oiseaux Gibiers Au Bénin
}

\author{
Avado C. Richard, Drs. \\ Département de Géographie et Aménagement du Territoire, \\ Université d'Abomey-Calavi (DGAT/UAC) \\ Lougbegnon O. Toussaint, $M C$ \\ Ecole de Foresterie Tropicale (EForT), \\ Université Nationale d'Agriculture (UNA), Kétou, Bénin
}

Gbesso G. H. François, MA

Ecole d'Horticulture et d'Aménagement des Espaces Verts (EHAEV),

Université Nationale d'Agriculture (UNA), Kétou, Bénin

Codjia Jean T. Claude, PT

Ecole de Foresterie et Tropicale (EForT),

Université Nationale d'Agriculture (UNA), Kétou, Bénin

Doi: 10.19044/esj.2018.v14n36p225 URL:http://dx.doi.org/10.19044/esj.2018.v14n36p225

Abstract
The two species of francolin used as game birds in Benin are
Francolinus albogularis Hartlaub and Francolinus bicalcaratus Linnaeus
belong to the family Phasianidae. This study aims to evaluate the importance
of the commercialization of these game birds at the level of the different actors
involved in the value chain. Data collection is based on structured and semi-
structured surveys conducted at the outlets of species along inter-state roads,
in restaurants and hotels, public dining places and bushmeat markets as well
than consumers. In total, 495 people were surveyed. The data collected is
essentially the actors of the marketing chain, the evolution of the price
variation of an individual of francolin, the different transaction circuits, the
places of supply. The Minitab 16 software, with the descriptive statistical
function, was used to assess the evolution of the price of a francolin individual
over the last thirty years among the actors of the marketing circuit. Calculated
meat supply frequencies and Kruskal-Wallis tests were performed. The results
from the analysis of the data show that three main actors intervene in the
distribution of the product, a varaiation of frequency of supply of the meat, the
price of sale of an individual of francolin rose from 50 FCFA there is thirty
years to 1600 FCFA this day at the level of hunters. For women processors, 
this price went from 200 FCFA twenty years ago to 1500 FCFA that day; while he spent for the same period from 300 FCFA to 3500 FCFA among the tenants of restaurants and hotels. This shows a consequent increase in prices. The Kruskal-Wallis test revealed a significant difference between the average selling prices of an individual francolin by hunters from thirty years ago, twenty years, ten years, five years ago and then today. It follows from all the above that urgent measures are being taken to adopt a domestication model for the multiplication of individuals and the valorization of francolins' meat.

Keywords: Francolinus albogularis Hartlaub, Francolinus bicalcaratus Linnaeus, bush meat, domestication, Benin

\section{Résumé}

Les deux espèces de francolin utilisées comme oiseaux gibiers au Bénin sont Francolinus albogularis Hartlaub et Francolinus bicalcaratus Linnaeus appartenent à la famille des Phasianidae. Cettte étude vise à evaluer l'importance de la commercialistion de ces oiseaux gibiers au niveau des différents acteurs intervenant dans la chaine de valeur. La collecte des données est basée sur les enquêtes structurée et semi-structurée conduites sur les points de vente des espèces situés le long des routes inter-Etat, dans les restaurants et hôtels, les lieux de restauration publics et les marchés de viande de brousse ainsi que les consommateurs. Au total, 495 personnes ont été enquêtées. Les données collectées sont essentiellement les acteurs de la chaine de commercialisation, l'évolution de la variation du prix d'un individu de francolin, les différents circuits de transaction, les lieux d'approvisionnement. Le logiciel Minitab 16., avec la fonction statistique descriptive, a servi à apprécier l'évolution du prix d'un individu de francolin les trente dernières années chez les acteurs du circuit de commercialisation. Des fréquences d'approvisionnement de viande calculé et des tests de Kruskal-Wallis ont été effectués. Les résultats issus de l'analyse des données montrent que trois princiâux acteurs interviennent dans la distribution du produit, une varaiation de fréquence d'approvisionnement de la viande, le prix de vente d'un individu de francolin est passé de 50 FCFA il y a trente ans à 1600 FCFA ce jour au niveau des chasseurs. Chez les transformatrices, ce prix est passé de 200 FCFA il y a vingt ans à 1500 FCFA ce jour ; alors qu'il est passé pour la même période de 300 FCFA à 3500 FCFA chez les tenanciers de restaurants et hôtels. Ceci montre une augmentation conséquente des prix. Le test de KruskalWallis a révélé une différence significative entre les prix moyens de vente d'un individu de francolin par les chasseurs d'il y a trente ans, vingt ans, dix ans, cinq ans passés et puis aujourd'hui. Il découle de tout ce qui précède que des mesures urgentes soient prises adopter un modèle de domestication en vue de la multiplication des individus et de la valorisation de la viande des francolins. 
Mots-clés: Francolinus albogularis Hartlaub, Francolinus bicalcaratus Linnaeus, viande de brousse, domestication, Bénin

\section{Introduction}

En Afrique et plus particulièrement en Afrique de l'Ouest, la faune est traditionnellement considérée par la population comme une propriété commune librement accessible et fait partout l'objet d'une consommation domestique et d'une exploitation économique. Les populations locales accordent une grande valeur à l'utilisation économique de la viande de brousse (Asibey, 1974 ; Ajayi, 1978).

$\mathrm{Au}$ Bénin, la croissante démographique très accélérée et les besoins vitaux afférents, la paupérisation grandissante avec pour conséquence une très forte exploitation alimentaire et commerciales des ressources forestières ont conduit les populations locales à intensifier le braconnage sans jamais égaler des espèces animales même les plus négligées par la chasse par le passé. Certaines espèces déjà vulnérables ou d'intérêt patrimonial font l'objet d'une chasse commerciale nationale et internationale comme en témoignent les marchés de vente d'animaux vivants ou morts dans les grandes villes du Bénin (Lougbégnon, 2015).

Actuellement, les marchés du Bénin, loin d'être spécialisés dans le commerce de la viande de brousse, contribuent potentiellement à cette activité qui a totalement transformé la chasse de subsistance traditionnelle en une activité purement commerciale : ce sont des lieux où le produit de la chasse (souvent à l'état frais ou cuit) est directement transformé en monnaies sonnantes et trébuchantes. Parmi les espèces de la faune avienne, toujours retrouvées sur les marchés béninois ou en transaction le long des axes routiers du pays, les espèces de francolins y occupent une place de choix. En effet, la viande de francolins est une source de protéines très appréciée par les consommateurs ruraux et urbains. La teneur en protéines brutes est très élevée et varie entre 81,3 et 91,3\% de matière sèche (Ekué, 2000). Toutes ces valeurs nutritives relevées de la viande de francolins contribuent à sa valeur économique élevée aux plans national et international (Aliou, 1986) en les rendant très vulnérables. A cet effet, les travaux d'imminents chercheurs béninois et nigérians comme Codjia et al. (2003), Ekué et al. (2002), Ajavi et al. (1973), Bah-Agban (2001) ; Idassa (1987) ; Mensah et al., (2001 et 2004) et Heymans (1973) se sont uniquement rapportés sur Francolinus bicalcaratus. Certains de ces auteurs tels que Ekué et al., (2002) et Aliou (1986) ont mentionné qu'au Sud-Bénin, la pression sur l'espèce est particulièrement très élevée en raison de sa valeur économique, de l'usage en médicinale 
traditionnelle de certains organes et son régime alimentaire qui est en compétition directe avec celui de l'Homme.

Actuellement, force est de constater que le commerce et la consommation des espèces de francolin ne se limite plus seulement à Francolinus bicalcaratus. Il se fait également et de manière très intense au détriment du Francolinus albogularis.

Cette recherche va permettre de créer une base scientifique solide sur l'importance de ces deux oiseaux gibiers en vue de leur conservation suivant une politique de domestication in situ.

\section{Matériel et Méthodes}

\section{Description du milieu d'étude}

La présente étude s'est déroulée sur toute l'étendue du territoire nationale située en Afrique de $1^{\prime}$ Ouest entre $6^{\circ} 10^{\prime}$ et $12^{\circ} 25^{\prime}$ de latitude nord et entre $0^{\circ} 45^{\prime}$ et $3^{\circ} 55^{\prime}$ de longitude est. Elle est limitée au Nord par le fleuve Niger, au Sud par 1'Océan atlantique, à 1'Est par la République Fédérale du Nigéria et à l'Ouest par les Républiques du Togo et du Burkina-Faso (figure 1) avec une superficie de $114.763 \mathrm{~km} 2$ (INSAE, 2008). Trois grandes zones climatiques (la zone guinéenne ou subéquatoriale, la zone de transition guinéo-soudanienne et la zone soudanienne) font la particularité géophysique du Bénin. La variation de la physionomie végétative est fonction des zones climatiques avec des types de sols caractéristiques ainsi que la faune. Les principales sources de revenus et de moyens de subsistance du monde rural sont l'agriculture, le commerce et la chasse. 


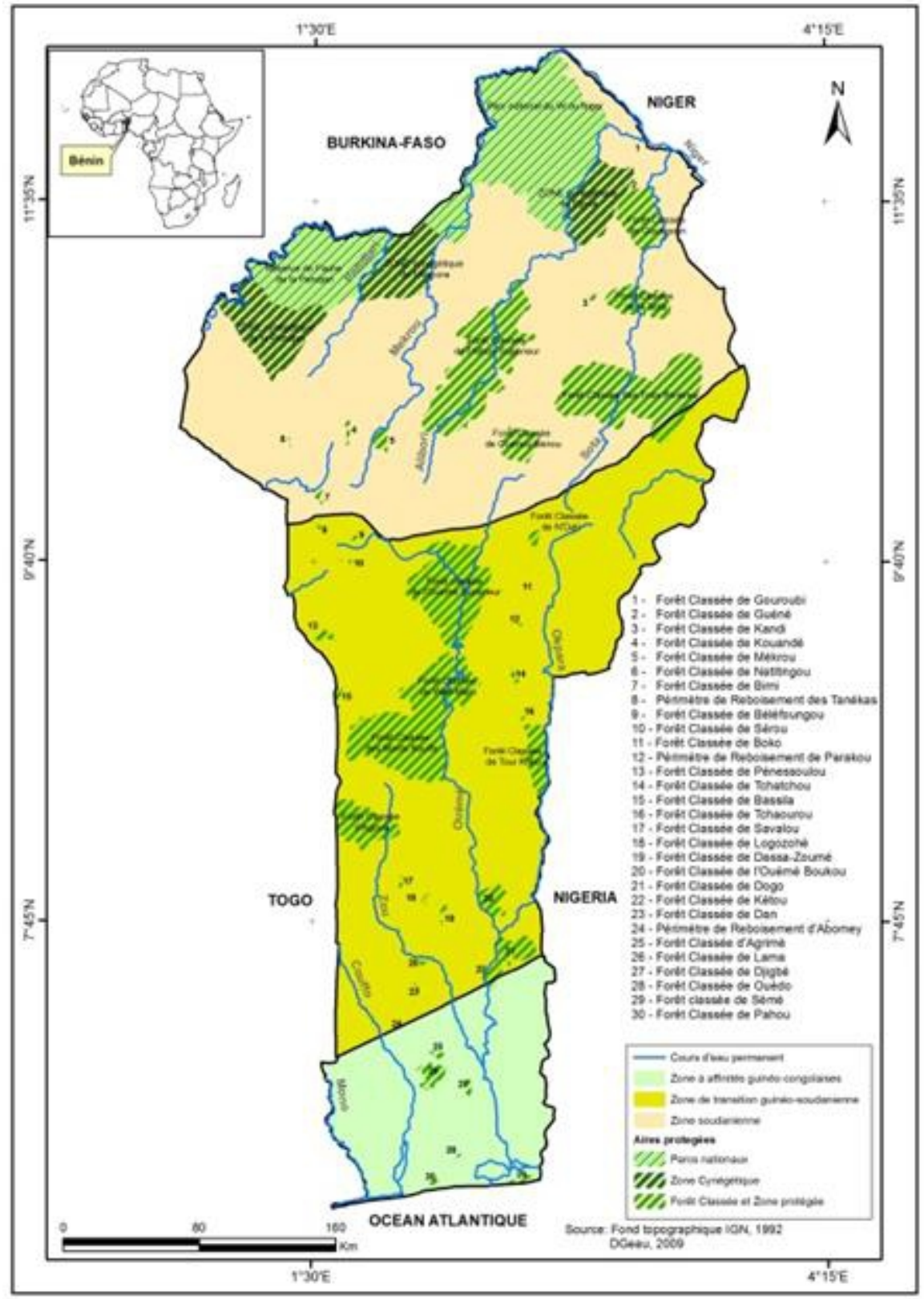

Figure 1 : Milieu d'étude

\section{Méthode de collecte des données}

Les variables collectées sont : l'identité des enquêtés, l'évolution les trente dernières années de la variation du prix d'un individu de francolin au niveau chasseurs, collecteurs, transformateurs, restaurants et hôtels, vendeurs dépouilles animales, l'évolution des modes de transformation, de stockage et de consommation ainsi que la valeur organoleptique de la viande, les différents 
circuits de transaction ou de commercialisation, la part des produits de chasse destinés à la vente et à la consommation familiale, les réalisations matérielles et immatérielles faites à partir de l'économie issue de la vente des francolins.

\section{Matériels d'étude}

Le matériel biologique utilisé est constitué de deux espèces Francolinus albogularis (Hartlaub) et Francolinus bicalcaratus (Linnaeus) quotidiennement utilisées par les populations et ayant une très forte valeur alimentaire et commerciale aux plans national et international.

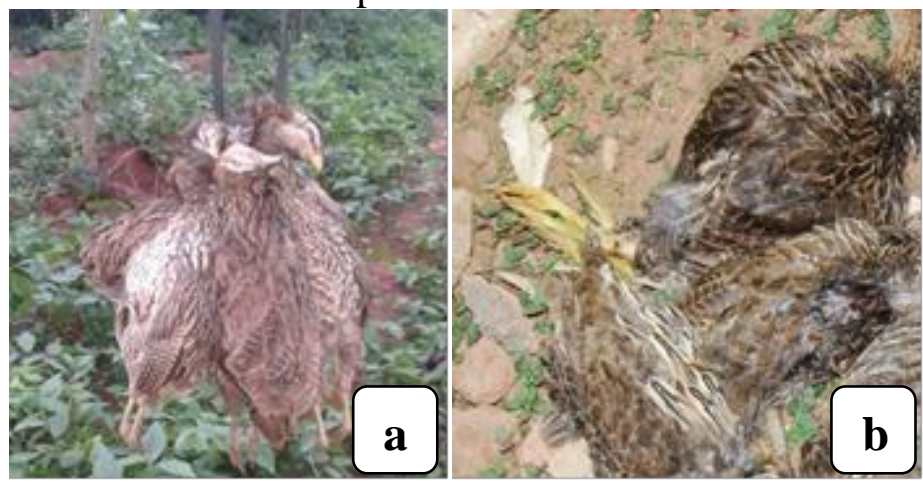

Photo 1 : Francolinus bicalcaratus (Linnaeus, 1766) capturé vivant (a) et Francolinus albogularis (Hartlaub, 1854) abattu (b)

L'habitat de Francolinus bicalcaratus est constitué par des champs de cultures vivrières, de jeunes jachères, les mosaïques de cultures et de jachères, les friches, les plantations, des galeries forestières très dégradées. Ces milieux constituent à la fois pour l'espèce, sa niche alimentaire (aire de pitance) et sa niche de dornance (endroit où il passe la nuit).

Les champs de cultures vivrières, les jeunes jachères, les mosaïques de cultures et de jachères, les friches et les galeries forestières très dégradées constituent la niche alimentaire pour Francolinus albogularis tandisque les savanes ouvertes en sont sa niche de dormance.

Le matériel technique comprend d'une part les fiches d'enquête et d'autre part les appareils portables de type androïde et tablettes de diverses marques ayant permis de prendre certaines vues et d'enregistrer certains témoignages très pertinents.

\section{Collecte des données}

La collecte des données a été faite auprès des chasseurs et collecteurs de viandes de brousse sur les marchés des villages, des villes, auprès des gérants des restaurants et hôtels, les transformateurs, les consommateurs et les vendeurs de dépouilles animales. La méthode du choix raisonné et du 
diagnostic participatif a permis le choix des différents acteurs. Au total, 495 personnes ont été enquêtées.

Les données collectées sont :

- les acteurs de la chaine de commercialisation de la viande de francolin ;

- l'évolution de la variation du prix d'un individu de francolin au niveau chasseurs, collecteurs, des transformateurs/transformatrices, des restaurants et hôtels, des acteurs de la restauration publique et des vendeurs de dépouilles animales durant les trente dernières années ;

- les différents circuits de transaction, de commercialisation et de distribution de la viande de francolin ;

- la fréquence d'approvisionnement (livraison par les chasseurs ou les collecteurs)

- les différents flux journaliers sur les axes d'approvisionnement, les zones de chasse, les villes de collecte et de consommation et les moyens de transport utilisés pour acheminer la viande de francolin vers les villes de consommation. La définition des flux consiste à compter pendant trois jours successifs, le nombre d'individus de francolins abattus qui transite par un axe routier donné ou dans une zone donnée.

\section{Analyse des données}

Une base de données a été réalisée sous tableur Excel, version 2016. Elle relie l'identité de chaque enquêté à ses différentes déclarations relatives aux variables collectées. Les formes de transformation, de commercialisation et de consommation de la viande de francolins ainsi que les réalisations matérielles et immatérielles ont été retenues à partir du score réel, basé sur la déclaration sans ambigüité d'au moins $51 \%$ des enquêtés. L'analyse de la variation de l'évolution du prix d'un individu de francolin sur les trente dernières années au niveau chasseurs, collecteurs, revendeurs ou transformateurs, hôtels et restaurants a été faite au moyen du logiciel Minitab, version 16.

La catégorisation des flux journaliers de commercialisation de la viande de francolin est appréciée selon la variation du nombre d'individus de francolin abattus et comptés dans chaque localité et sur chaque axe routier. Ainsi, on a des flux:

- locaux ( si le nombre journalier d'individus de francolins comptés varie de 5 à 10);

- faible (si le nombre journalier d'individus de francolins comptés varie de 10 à 20);

- moyennement élevé (si le nombre journalier d'individus de francolins comptés varie de 21 à 50);

- élevé (si le nombre journalier d'individus de francolins comptés varie de 51 à 100); 
- très élevé (si le nombre journalier d'individus de francolins comptés est supérieur à 100).

\section{Résultats}

\section{Acteurs intervenant dans la distribution de la viande de francolins}

Le circuit de transaction et de commercialisation de la viande du francolin est structuré en quatre grandes catégories d'acteurs que sont les chasseurs, les collecteurs, les transformateurs et les consommateurs du produit fini. Les chasseurs et les collecteurs qui forment un véritable contingent dans le domaine. Les transformatrices ambulantes sont pour la plupart très outillées et très attentives quant aux exigences des clients en matière de transformation de la viande du francolin. Les détenteurs de restaurants et hôtels sont très efficaces pour donner tous les goûts exigés par la clientèle. La concurrence entre ces acteurs se traduit par leur capacité à se particulariser dans la transformation et la diversification des modes de préparation de la viande du francolin. L'ensemble de tous ces acteurs sont très organisés en réseau de distribution et de commercialisation de la viande de francolin (figure 2). Le niveau de l'organisation du marché de viande de francolin et la dynamique des acteurs à alimenter les différents points de transformation et de consommation de cette viande permettent de parler de la 'filière francolin', au Bénin.

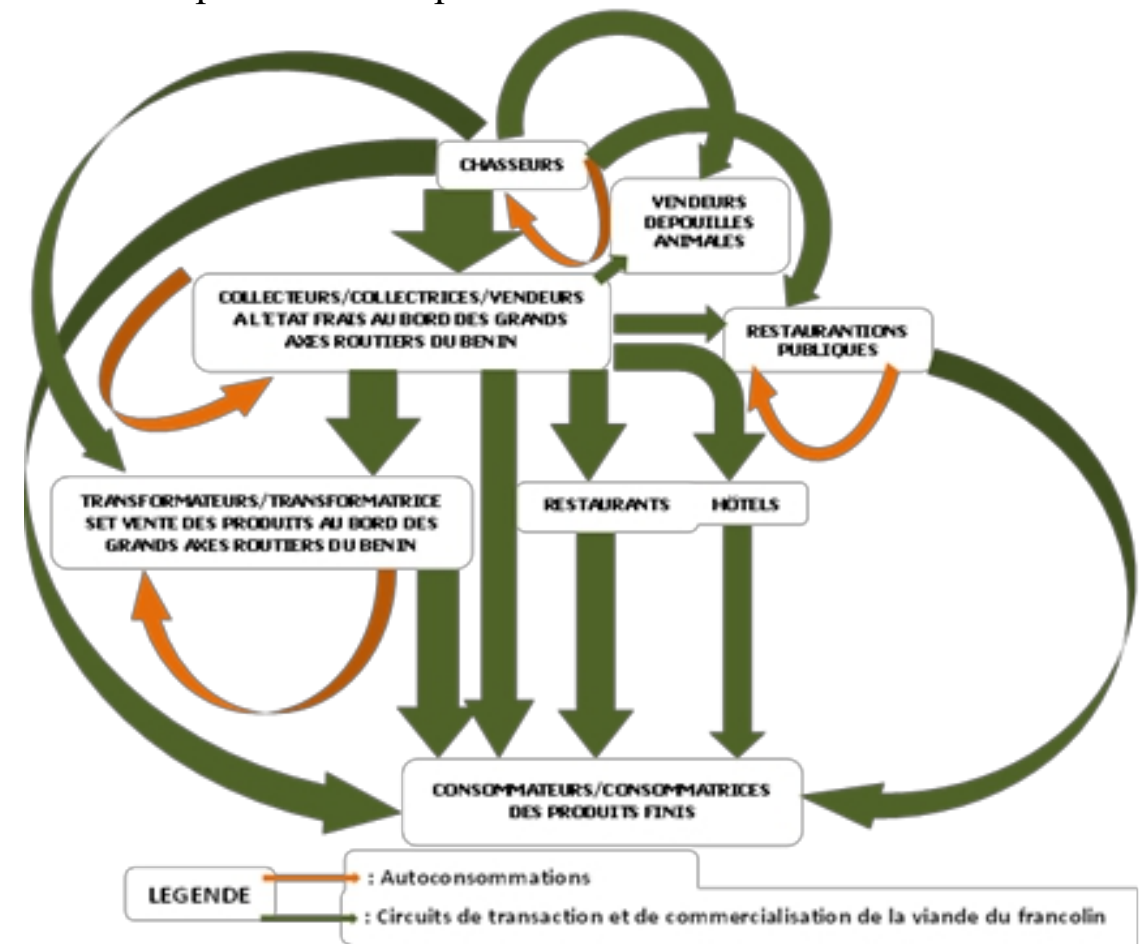

Figure 2 : Circuit de distribution et de commercialisation de la viande du francolin 
Chaque acteur du circuit de distribution de la viande de francolin se reconnait un rôle bien précis et bien déterminé :

- les chasseurs : ce sont les acteurs spécialisés en matière de capture des espèces de francolin. Certains chasseurs se déplacent vers les collecteurs ou les transformatrices ambulantes sous le contrat que les frais liés à leur transport sont remis sur le montant de vente du nombre d'individus de francolins livrés. D'autres chasseurs par contre, reçoivent à leur domicile les collecteurs et/ou les transformatrice ambulantes pour leur livrer le produit de chasse. Pour 98, $77 \%$ de cette catégorie d'acteur, $90 \%$ voire $100 \%$ du produit de chasse est destiné à la vente. Seulement $10 \%$ ou bien zéro pour cent de produit de chasse est orienté à la consommation familiale.

- les collecteurs : ils sont très actifs sur le terrain et se livrent par moments entre eux de rudes concurrences. Chaque collecteur de francolin abattu est en réseau avec plusieurs chasseurs. La disponibilité de produits de chasse leur est signalée par un simple appel téléphonique. Les produits de chasse collectés en occurrence les francolins sont distribués aux transformatrices ambulantes et aux tenanciers de restaurants ou hôtels ainsi que les bonnes dames de la restauration publique.

- les transformateurs : ils sont de plusieurs catégories. On peut citer les transformatrices ambulantes (exemple des transformatrices vendeuses de la viande de francolin au bord de la RNIE nº 2 à Allada et à Sèhouè ou installées dans les maisons à Houègbo), les transformatrices dans les lieux de restaurations publiques à travers tout le pays et les transformateurs des restaurants et hôtels.

\section{Fréquence d'approvisionnement en viande}

La fréquence moyenne hebdomadaire d'approvisionnement en viande de francolin varie d'un acteur de transformation de la viande à un autre. En effet, les transformatrices ambulantes s'approvisionnent tous les jours ( 7 jours sur 7 jours) en viande de francolin tandis que les détenteurs des lieux de restauration publique s'en approvisionnent en moyenne 5 jours sur 7 jours par semaine. Quant aux détenteurs des restaurants et hôtels, la fréquence hebdomadaire moyenne d'approvisionnement en viande de francolin est de 3 jours sur 7 jours (figure 3). Selon 97,59\% de ces derniers acteurs, les conditions efficaces et sécurisées de conservation de la viande des espèces de francolin justifient cette faible fréquence d'approvisionnement : le stock de la viande de francolin réalisé lors d'un approvisionnement est conservé au congélateur afin de satisfaire régulièrement les clients. 


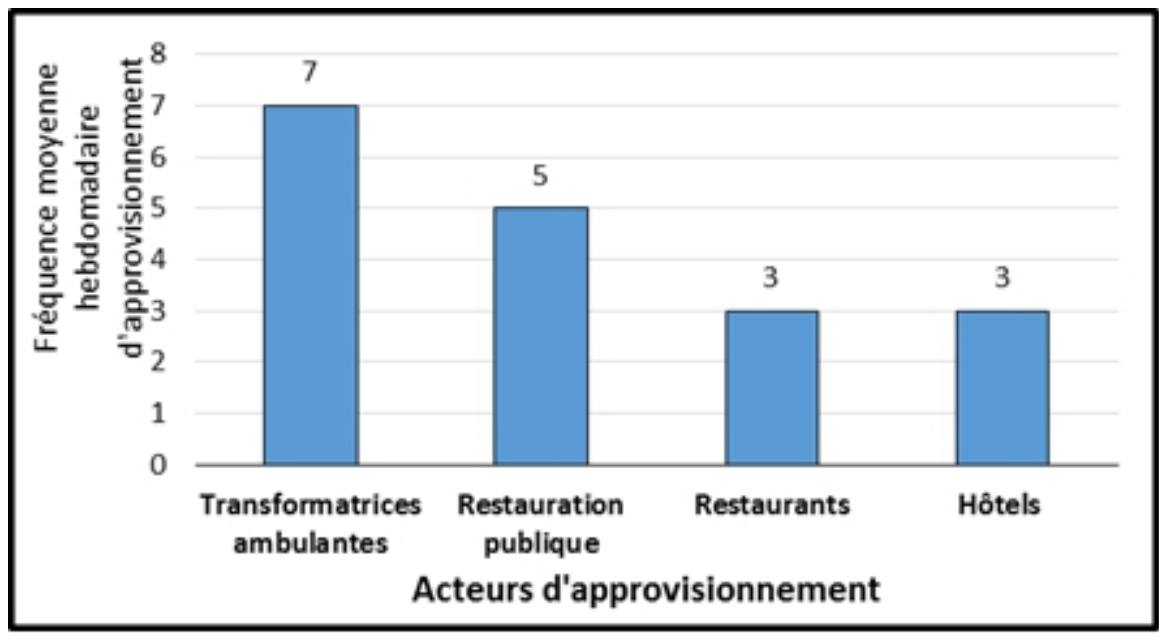

Figure 3 : Fréquence moyenne hebdomadaire d'approvisionnement

\section{Axes de ravitaillement en viande de francolin et villes de convergence}

Les principaux axes utilisés pour assurer le ravitaillement des grandes villes en viande de francolin sont les routes nationales avec comme principal moyen de transport les différents bus des diverses compagnies de voyage qui assurent la liaison Sud-Nord dans le pays.

Pour réussir le commerce de la viande de francolin, plusieurs villes situées le long des grands axes routiers constituent des zones de collecte des individus de francolin prélevés par la chasse dans les zones rurales avant d'être acheminé vers les grands centre de consommation. Ce sont des villes comme Natitingou, Djougou, Bassila, Kandi, N'Dali, Parakou, Tchaourou, Dassa, Bohicon, Sèhouè, Houègbo, Allada et Pobè. Les plus importants centres de collecte sont les villes de Natitingou, de Djougou, de Parakou, de Dassa et de Bohicon tandis que le centre le moins important est la ville de Comè. Certaines de ces villes assurent d'autres fonctions capitales (transformation et vente sur place) que celle relative à la collecte de la viande de francolin. Il s'agit de Sèhouè, Houègbo et Allada. Les villes de Cotonou, de Sèmè-Podji et de PortoNovo sont les grands centres de consommation de la viande de francolin au Bénin.

Par ailleurs, le flux d'approvisionnement de la viande de francolin est très élevé à partir de Natitingou en passant par Djougou, Bassila, Dassa, Bohicon jusqu'à Cotonou. Il est élevé de Parakou jusqu'à Cotonou et de Kétou jusqu'à Porto-Novo, Sèmè-Podji et à Cotonou en passant par Pobè. Ce flux d'approvisionnement reste faible pour le reste du Pays (figure 4). 


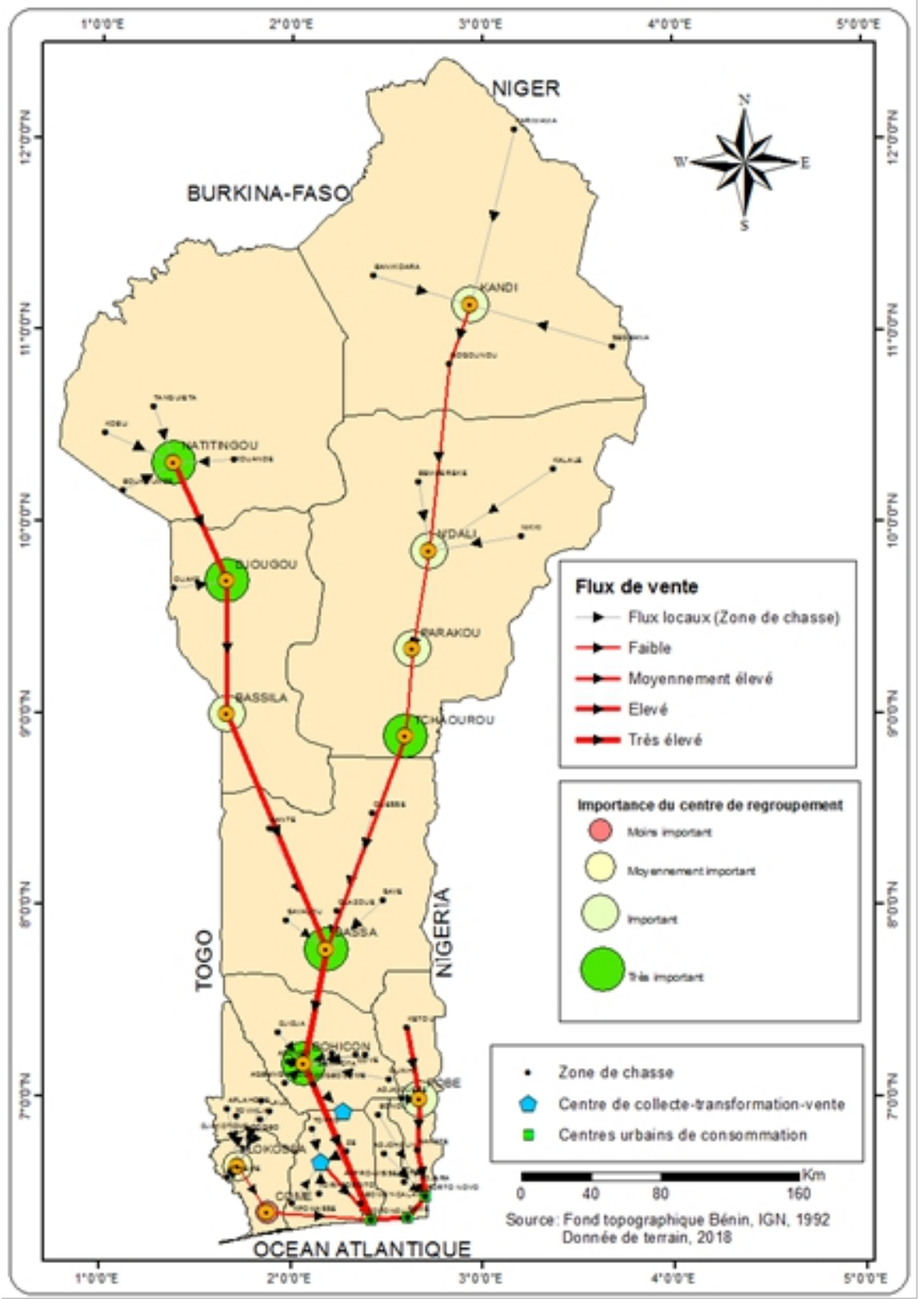

Figure 412: Axes d'approvisionnement et centres de collecte, de transformation et de consommation de la viande de francolin

\section{Valeur économique commercialisation de la viande du francolin} Evolution du prix d'un individu de francolin chez les chasseurs

Il ressort des résultats issus de l'analyse des données collectées auprès de cette catégorie d'acteur que le prix de vente d'un individu de francolin a évolué dans le temps et dans l'espace. En effet, il y a trente ans, le prix minimum pour acheter un individu de francolin chez un chasseur varie 50 et 
250 FCFA. Par contre, actuellement, il varie entre 400 FCFA et 1600 FCFA (tableau 1).

Tableau 1 : Variation du prix de vente entre chasseurs et collecteurs

\begin{tabular}{|c|c|c|c|c|}
\hline & \multicolumn{4}{|c|}{ Prix de vente } \\
\hline Période & $\begin{array}{c}\text { Prix moyen } \\
\text { (FCFA) }\end{array}$ & $\begin{array}{c}\text { Coefficient de } \\
\text { variation }(\%)\end{array}$ & $\begin{array}{l}\text { Prix minimum } \\
\text { (FCFA) }\end{array}$ & $\begin{array}{c}\text { Prix maximum } \\
\text { (FCFA) }\end{array}$ \\
\hline 30ans_passé & 140,51 & 30,16 & 50 & 250 \\
\hline 20ans_passé & 262,89 & 35,02 & 125 & 700 \\
\hline 10ans_passé & 373,86 & 34,19 & 200 & 1000 \\
\hline 5ans_passé & 529,72 & 33,33 & 250 & 1200 \\
\hline Actuel & 840,67 & 30,58 & 400 & 1600 \\
\hline
\end{tabular}

Le prix moyen de vente d'un individu de francolin par les chasseurs a connu une évolution ascendante depuis trente ans à ce jour. Le test de KruskalWallis a révélé une différence significative entre les prix moyens de vente d'un individu de francolin par les chasseurs d'il y a trente ans, vingt ans, dix ans, cinq ans passés et puis aujourd'hui (figure 5).

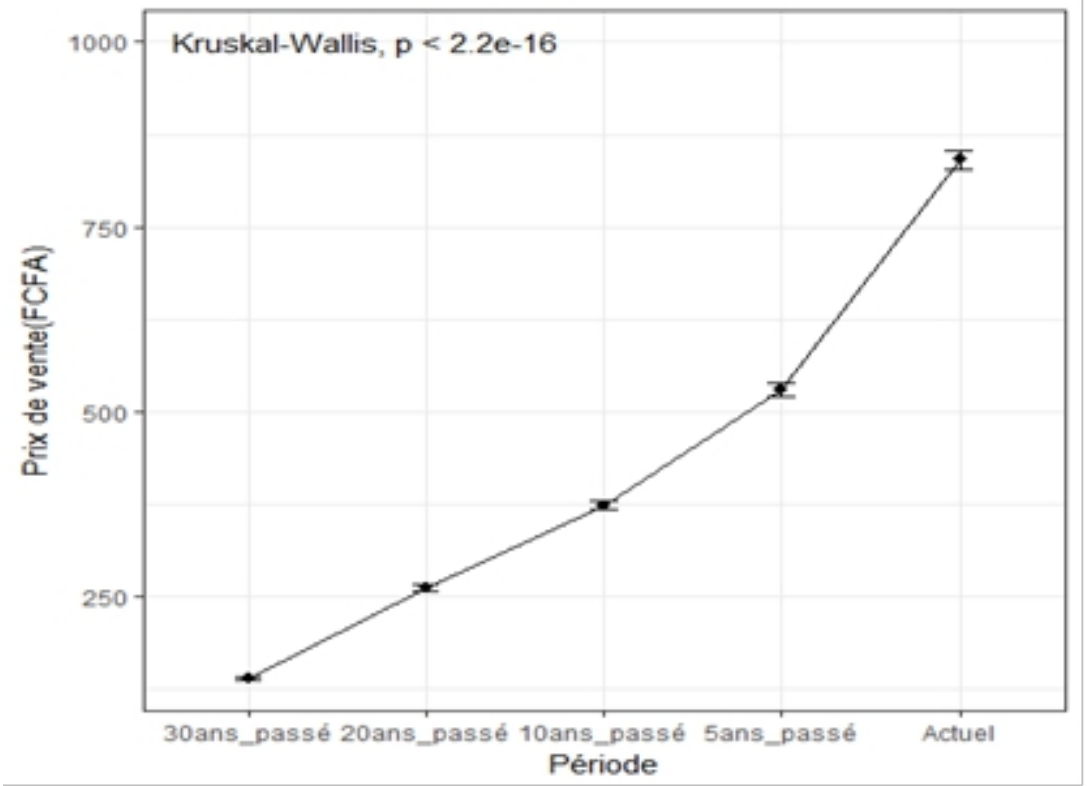

Figure 5 : Evolution du prix de vente d'un individu de francolin chez les chasseurs

Evolution du prix d'achat et de vente d'un individu de francolin chez les transformatrices ambulantes

Les résultats de l'analyse des données collectées auprès des acteurs révèlent que le prix d'achat d'un individu de francolin auprès des receveurs/collecteurs a aussi évolué dans le temps et dans l'espace chez les transformatrices ambulantes. En effet, il y a vingt ans, il leur faut au minimum 200 FCFA et au maximum 400 FCFA pour se procurer un individu de francolin. Aujourd'hui, elles déboursent entre 1000 et 1200 FCFA pour 
pouvoir acquérir un individu de francolin puis les vendent entre 1000 et 1500 FCFA après l'avoir transformé (tableau 2).

Tableau 2: Variation des prix d'achat et de vente d'un francolin chez les transformatrices

\begin{tabular}{c|ccccc}
\hline Périodes & $\begin{array}{c}\text { Nature du } \\
\text { prix }\end{array}$ & $\begin{array}{c}\text { Prix moyen } \\
\text { (FCFA) }\end{array}$ & $\begin{array}{c}\text { Coefficient } \\
\text { de } \\
\text { Variation }\end{array}$ & $\begin{array}{c}\text { Prix } \\
\text { minimum } \\
\text { (CFA) }\end{array}$ & $\begin{array}{c}\text { Prix } \\
\text { maximum } \\
\text { (CFA) }\end{array}$ \\
\hline 20ans_passé & PrixAchat & 323,48 & 15,91 & 200 & 400 \\
20ans_passé & PrixVente & 555,33 & 15,77 & 400 & 700 \\
10ans_passé & PrixAchat & 539,63 & 23,60 & 300 & 700 \\
10ans_passé & PrixVente & 797,58 & 13,17 & 550 & 950 \\
5ans_passé & PrixAchat & 868,32 & 7,42 & 650 & 950 \\
5ans_passé & PrixVente & 939,40 & 7,15 & 800 & 1000 \\
Actuel & PrixAchat & 1037,10 & 6,80 & 1000 & 1200 \\
Actuel & PrixVente & 1470,74 & 7,75 & 1000 & 1500 \\
\hline
\end{tabular}

Les prix d'achat et de vente d'un individu de francolin par les transformatrices ambulantes ont corrélativement évolué depuis vingt ans à cinq ans passés, année à partir de laquelle le prix de vente a brusquement augmenté jusqu'à ce jour. Le test de Kruskal-Wallis a révélé une différence significative entre la variation des prix d'achat et de vente d'un individu de francolin il y a vingt ans et aujourd'hui (figure 6).

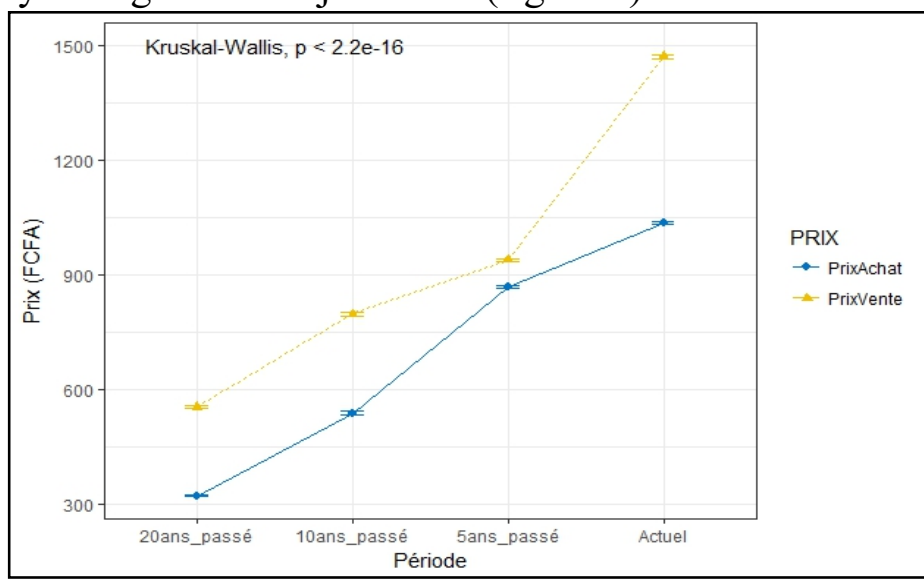

Figure 6 : Evolution comparée du prix d'achat et de vente d'un individu de francolin chez les transformatrices ambulantes

\section{Evolution du prix d'un individu de francolin dans les restaurants et hôtels}

Les informations collectées auprès de ces acteurs indiquent que le prix d'achat et de vente d'un individu de francolin a également évolué dans les hôtels et restaurant dans le temps et dans l'espace. En effet, il y a vingt ans, les hôteliers et les détenteurs de restaurant achetaient un individu de francolin à 300 FCFA au minimum et à 600 FCFA au maximum. Aujourd'hui, il leur 
faut au minimum 1400 FCFA et au maximum 2000 FCFA pour acheter un francolin et le revendre au minimum à 2500 FCFA ou au maximum à 3500 aux consommateurs finaux après l'avoir transformé (tableau 3 ).

Tableau 3 : Variation des prix d'achat et de vente d'un individu de francolin dans les restaurants et hôtels

\begin{tabular}{cccccc}
\hline Période & $\begin{array}{c}\text { Nature du } \\
\text { prix }\end{array}$ & $\begin{array}{c}\text { Prix } \\
\text { moyen } \\
\text { (FCFA) }\end{array}$ & $\begin{array}{c}\text { Coefficient } \\
\text { de Variation }\end{array}$ & $\begin{array}{c}\text { Prix } \\
\text { minimum } \\
\text { (FCFA) }\end{array}$ & $\begin{array}{c}\text { Prix } \\
\text { maximum } \\
\text { (FCFA) }\end{array}$ \\
\hline 20ans_passé & PrixAchat & 456,52 & 22,47 & 300 & 600 \\
& PrixVente & 1318,18 & 20,76 & 900 & 1900 \\
10ans_passé & PrixAchat & 1156,52 & 11,61 & 1000 & 1300 \\
& PrixVente & 2081,82 & 4,83 & 2000 & 2200 \\
5ans_passé & PrixAchat & 1408,70 & 7,69 & 1200 & 1500 \\
& PrixVente & 2740,91 & 9,12 & 2500 & 3500 \\
& PrixAchat & 1513,04 & 8,76 & 1400 & 2000 \\
& PrixVente & 3327,27 & 16,34 & 2500 & 3500 \\
\hline
\end{tabular}

Par ailleurs, le test de Kruskal-Wallis a révélé une différence significative entre l'évolution comparée des prix de vente et d'achat des francolins suivant les périodes (figure 7).

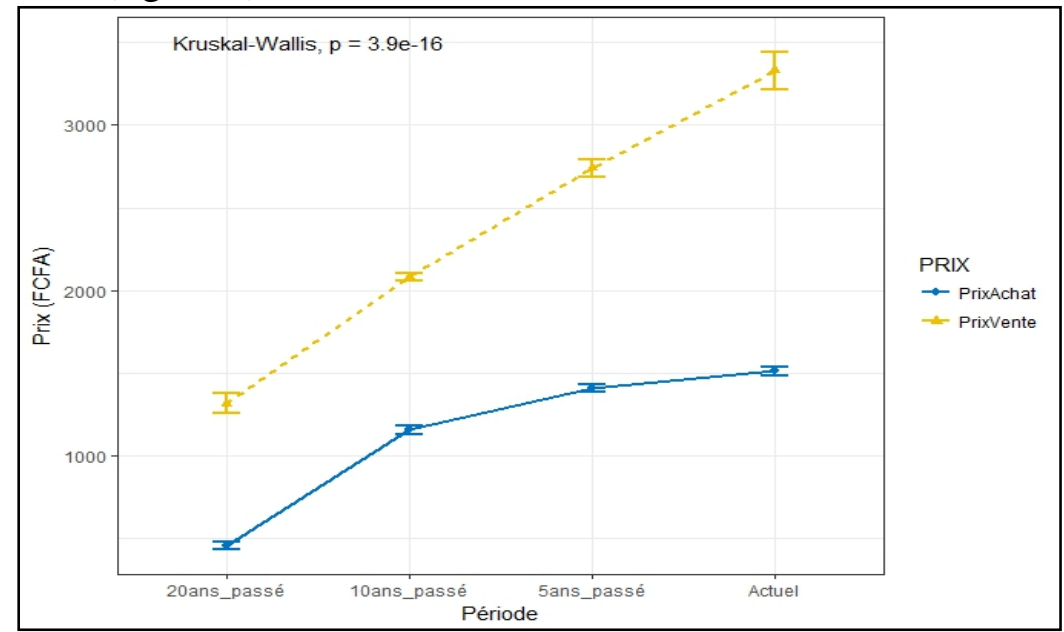

Figure 7 : Evolution comparée du prix d'achat et de vente d'un individu de francolin dans les restaurants et hôtels du Bénin

\section{Discussion}

Les résultats de cette recherche ont montré que le commerce de la viande des francolins comme viande de brousse génère des revenus très importants pour les différents acteurs qui oeuvrent pour faire du commerce de francolin, une filière à promouvoir. Ce constat concorde les travaux de Roger et al., 2011 qui mentionnait que la contribution de la viande de chasse dans l'économie des ménage est très significative. En effet, l'évolution de la forte 
demande provenant des centres urbains ont conduit à la naissance d'une chasse commerciale dont le but est de satisfaire les besoins exprimés par les populations urbaines et périurbaines, notamment l'introduction de la viande des francolins dans les ménus des restaurants et hôtels de grandes villes du Bénin. Ceci a conduit les acteurs à se mettre en réseaux pour faciliter la transaction de la viande de francolin. Ainsi, l'ampleur notée dans le commerce de la viande des francolins s'explique par le fait que les jeunes chasseurs, motivés par la monnaie sonnante et trébuchante découlant du commerce de la viande, destinent 90 à $100 \%$ des produits de chasse à la vente et le reste éventuel à la consommation familiale. Ce résultat contrarie les travaux de Heymans (1973) qui indiquaient que les chasseurs au Sud-Bénin destinent seulement $25 \%$ des francolins abattus à la vente et $75 \%$ à la consommation familiale. Cette inversion de tendance notée dans cette étude s'explique par l'augmentation rapide de la valeur monnétaire et commerciale de la viande du francolin qui constitue actuellement une source sûre de revenu pour les populations. Aussi, faut-il remarquer que le mode de vie extraverti des populations locales a conduit les jeunes chasseurs à opérer une pression sans jamais égalée sur la faune qu'ils destinent la quasi-totalité des produits de chasse à la vente pour s'acheter les télévisions avec décodeurs, les motos, les portables androïdes voire les tablettes, etc. Ces bien de luxe se sont ajoutés aux dépenses traditionnelles relatives à l'organisation des dots, les cérémonies coutumières et la construction des maisons.

La présente étude a également montré que le réseau routier contribue potentiellement à la commercialisation de la viande de francolin au Bénin. En effet, l'ouverture de voies de communication opérée par l'Etat et les collectivités locales favorisent l'acheminement assez rapide des produits de chasse des localités vers les grandes zones de collecte pour approvisionner les grandes villes qui sont des zones de fortes consommations de viandes de brousse au Bénin. Ce résultat corrobore avec les travaux de Codjia et Assogbadjo, 2004 et East et al., 2005 qui montraient que les habitants des zones rurales ayant comme principale source de revenu,la chasse, profitent de l'ouverture des pistes ruraux opérés par les sociétés forestières pour transporter leurs produits de chasse des points de collecte vers les grandes grandes villes et agglomérationsn des pays. Ainsi, la commercialisation de la viande du francolin a donné naissance dans le pays à des zones spécifiques de collecte telles que Natitingou, Djougou, Bassila, Kandi, N'Dali, Parakou, Tchaourou, Dassa, Bohicon, Sèhouè, Houègbo, Allada et Pobè. Toutes ces zones de collecte citées sont des plateformes à divers degré d'importance dans la commercialisation de la viande de francolin. Le principal facteur qui permet à ces villes d'être la plateforme incontournable dans le commerce de la viande de francolin est le réseau routier. En effet, toutes ces villes identifiées sont parcourues par les principaux axes routiers du Bénin. Par exemple, des 
marchés de viandes de brousse sont principalement créés à Allada, Sèhouè et Tchaourou le long de la RNIE n² Cotonou-Niger. Par ailleurs, le francolin abattu est vendu le long de tous les principaux axes routiers du pays au niveau des agglomérations. Ce résultat corrobore partiellement avec les résultats des travaux de recherche de Aliou, 1986 qui identifiait la ville de Pobè comme un centre commercial de la viande de francolin. Le fait que l'auteur n'avait pas signalé les autres villes et points de vente identifiés dans cette étude comme centres commerciaux de la viande de francolin pourrait se justifier par une généralisation du commerce de la viande de francolin dans dans le temps et dans l'espace.

\section{Conclusion}

L'utilisation alimentaire et économique des francolins a donné naissance à un circuit bien nourri de distribution et de commercialisation de la viande de francolin. Ce circuit met en relation très étroite les acteurs tels que les chasseurs, les collecteurs, les transformatrices, les vendeurs ou vendeuses à la sauvette, les détenteurs de restaurants, bars et hôtels et aussi les détenteurs des restaurations publiques, sans occulter les vendeurs de dépouille animale. Les principaux axes routiers qui favorisent l'approvisionnement de la viande du francolin sont notamment les RNIE avec pour villes de collecte Natitingou, Djougou, Bassila, Dassa, Kandi, N'Dali, Parakou et Pobè ainsi que des villes de collecte et de transformation comme Bohicon, Sèhouè, Houègbo, et Allada. Les principales villes où la viande de francolin est fortement consommée sont Cotonou, Sèmè-Podji, Porto-Novo et Abomey-Calavi.

La viande de francolin génère des revenus pour différents acteurs, notamment ceux qui prélèvent ; ceux qui transportent ou vendent la viande dans différents points de la chaîne d'approvisionnement : le long des routes, au marché, dans les restaurants et hôtels. Depuis le prélèvement initial jusqu'à la vente finale, le commerce de la viande de francolin destinée au marché local, national ou régional constitue désormais une partie importante de la filière « viande de brousse » qui participe activement à la vie de la société.

L'étude a permis de se rendre compte de la valeur économique des francolins et la pression exercée sur la survie des deux espèce. Alors il est impérieux de définr une politique de domesticatication de ces espèce en vue de leur durabilité.

\section{References:}

1. Adam K. S. et Boko M. Le Bénin. Edicef, Paris, 1993

2. Ajayi S.S., Arubayi, E. E. Food habits of francolin (Francolinus bicalcaratus) in Ibadan District. The Nigerian journal of forestry 3 (2) 51-58. 1973. 
3. Akande M. Bush fowl (Francolinus bicalcaratus bicalcaratus LINNAEUS (1766) as a pest and potential source of meat in Nigeria. Ph.D thesis. University of Ibadan. 176 p. 1979.

4. Aliou M. D., Contribution à l'étude écoéthologique du francolin (Francolinus bicalcaratus L.) en milieu naturel et son utilisation dans l'alimentation humaine. Thèse d'ingénieur Agronome, Université Nationale du Bénin, Faculté des Sciences Agronomiques d'AbomeyCalavi, 127 p. 1986.

5. Bah-Agban L. Francoliniculture : élevage du francolin commun (Francolinus bicalcaratus Linnaeus, 1766): alimentation en captivité étroite. Mémoire, DPA/CPU/ UNB/ Abomey-Calavi, 65 p. 2001.

6. Codjia, J.T.C., Ekué M.R.M., Mensah G. A., Ecologie du francolin à double éperon Francolinus bicalcaratus dans le Sud-Est du Bénin. Malimbus 25, 77-84.Conservation 143, 1493-1500. 2003.

7. Codjia, J.T.C. and Assogbadjo, A.E, Mammalian Wildlife as food supply resource for the rural population in the Lama Forest Reserve (South Benin). Cahiers Agricultures 13: 341-7. 2004.

8. East, T., Kümpel, N.F., Milner-Gulland, E.J., and Rowcliffe, J. M., Determinants of urban bushmeat consumption in Rio Muni, Equatorial Guinea. Conservation Biology 126(2): 206-215. 2005.

9. Ekué M. R. M. Etude Ecologique du Francolin commun (Francolinus bicalcaratus Linnaeus, 1766) et élaboration d'un référentiel pour son élevage en captivité étroite. Thèse d'ingénieur agronome. FSA/UNB, 2000.

10. Ekué, M.R.M., G.A. Mensah, Codjia J.T.C. Technique de capture du francolin commun (Francolinus icalcaratus Linnaeus, 1766) par usage du filet. In TROPICULTURA, 2002, Vol 20. N¹, Pp 44-48. 2002.

11. Ekué M. R. M., Mensah G. A. et Codjia J. T. C. Rendement en carcasse, rendement en viande et composition de la viande de francolin commun (Francolinus bicalcaratus L.). «Bulletin de la Recherche Agronomique du Bénin », n43, P 23-29. 2004.

12. Heymann, J. C., Utilisation de la viande de chasse et élevage de certaines espèces animales au Zaïre et au Bénin. Trop. Ans. Prod. for the benefit of Man. Int. Colloquium-Ant werpen Doc. 17-18 pp. 267273. 1973.

13. Idassa A., Ethologie du francolin commun (Francolinus bicalcaratus) dans la savane boisée du district rural de Tchaourou en République Populaire du Bénin. Rapport de stage. Ecole pour la formation des spécialistes de la faune. Garoua, Cameroun, 20 p. 1987.

14. INSAE, Quatrième Recensement Général de la Population et de l'habitant du Bénin, Résultats définitifs, Juin 2015, 35p. 2015. 
15. Lougbégnon, O. T., Ecologie et connaissances ethnozoologiques de quelques espèces d'oiseaux gibiers menaces des écosystèmes du Sud du Bénin. Thèse de doctorat en Biologie des Organismes et Ecologie, Université de Liège, 2015.

16. Mensah S. E. P. et Mensah G. A. Actogramme du francolin commun (Francolinus bicalcaratus bicalcaratus) élevé en captivité étroite. Bulletin de la Recherche Agronomique du Bénin (BRAB). Numéro spécial Elevages de gibier \& non gibier. Mai 2012, 9-22 P.Ecology 6: 14. 2012.

17. Mensah, G.A., Bah-Agban, L., Alimentation du francolin commun (Francolinus bicalcaratus) élevé en captivité étroite. In Programme et résumés/abstracts du 2ème Atelier scientifique Sud-Centre du Bénin. INRAB/FSA-UAC/SNRA Bénin/GTZ, CRA-Sud du Bénin - Niaouli, 12-13/12/2001. 2001.

18. Roger Albert Mbete, Henri Banga-Mboko, Christophe Ngokaka, Quevin Farège Bouckacka, Innocent Nganga, Jean-Luc Hornick, Pascal Leroy, Cédric Vermeulen, Profil des vendeurs de viande de chasse et évaluation de la biomasse commercialisée dans les marchés municipaux de Brazzaville, Congo. Tropical Conservation Science Vol.4 (2): 203-217. 2011. 